\title{
Modeling of polarization charge in N-face InGaN/GaN MQW solar cells
}

\author{
R. Belghouthi ${ }^{\text {a,* }}$, S. Taamalli ${ }^{\text {a,b }}$, F. Echouchene ${ }^{a}$, H. Mejri ${ }^{\text {a }}$, H. Belmabrouk ${ }^{\text {a }}$ \\ a Laboratoire d'Electronique ET de Microélectronique, Département de physique, Faculté des Sciences de Monastir, 5019, Tunisia \\ ${ }^{\mathrm{b}}$ Laboratoire de Photonique d'anger, E.a. 4464, Université d'anger, 2Bd Lavoisier, 49045 Angers Cedex 01, France
}

\section{A R T I C L E I N F O}

\section{Article history:}

Received 14 May 2015

Received in revised form

6 July 2015

Accepted 6 July 2015

Available online 16 July 2015

Keywords:

InGaN/GaN MQW solar cells

Spontaneous and piezoelectric polarizations

Conversion efficiency

\begin{abstract}
A B S T R A C T
The present work reports on a theoretical study of spontaneous and piezoelectric polarization effects on the photovoltaic characteristics of InGaN/GaN multiple quantum well solar cells. More especially, it will prove that the use of heterostructures with $\mathrm{N}$-face as a surface polarity can further improve the photovoltaic conversion. A new model including piezoelectric polarization is developed. In this paper, a part of simulation is also paid to analyze the dependence of the photocurrent density, the open circuit voltage, the output power and the efficiency versus the In composition and the number of quantum well units. As has been found, a maximum of energy conversion is expected to achieve 19 percent for optimum alloy composition. An attempt to explain the photovoltaic behavior of the solar cells in correlation of obtained results will be attempted.
\end{abstract}

(c) 2015 Elsevier Ltd. All rights reserved.

\section{Introduction and related works}

The design and manifacturation of optoelectronic devices may take advantage of piezoelectric properties of some semiconductors such as GaN and InN and their alloys. Indeed, III-nitride materials have wide band gaps, large breakdown bias voltages and strong spontaneous and piezoelectric polarization fields as well as an efficient electron transport [1]. Due to polarization, a two-dimensional electron gas (2DEG) can occur at hetero-interfaces with relatively high densities even without an intentional doping [2]. In solar cell applications, III-nitrides have recently emerged as promising candidates mainly due to their tunable energy band gaps and photovoltaic characteristics $[3,4]$.

For InGaN, the band gap can vary continuously from ultraviolet at $3.42 \mathrm{eV}$ to the near infrared at $0.77 \mathrm{eV}$, which matches well with the whole solar spectrum. Additionally, InGaN-related systems show many other favorable properties including high absorption coefficients, high carrier mobility, saturation velocities, and a superior radiation resistance [5,6].

However, there are several limiting factors that can affect the photovoltaic conversion. The main critical issue consists in high In incorporation and inadequate controlling during epitaxial growth of thin InGaN films. The ideal indium composition for high efficiency InGaN is expected to range from 0.6 to $0.7[7,8]$. However, the growth of InGaN/GaN with relatively high indium compositions is difficult due to high lattice mismatch between GaN and

\footnotetext{
* Corresponding author.

E-mail address: rabebbelghouthi@gmail.com (R. Belghouthi).
}

InGaN. The lattice mismatch together with low temperature growth of InGaN can introduce many defects, which lead to the reduction of carrier lifetime and thus diminish the photocurrent [9].

Moreover, the InGaN epitaxial layers suffer from both spontaneous and piezoelectric polarization fields [10,11]. Due to this limiting factor, the influence of polarization on the performance of solar cells based on the InGaN/GaN system has been extensively investigated and was the subject of many reports [12-18].

In Ga-face GaN/ InGaN, the polarization charges have a detrimental effect on the solar cell performance due to the increasing of energy barrier heights for holes. The induced electric field at the GaN/InGaN interface reinforces the photo-created carriers to drift in opposite direction of the efficient collection and substantially reduces both the short circuit current and the open circuit voltage $[9,12]$. To address those problems, several alternatives have been proposed as promising solutions, like the incorporation of a linearly graded junction between GaN and InGaN or the use of homojunction structures [9-16].

In the present work, we propose a new design of solar cells by using of InGaN/GaN MQW hetero-structures with $\mathrm{N}$ face polarity. The reason is that this architecture eliminates the hole barrier and forces free carriers to drift towards the efficient collection which enhances the photo current $[17,18]$.

The aim of this paper is dedicated to investigate the photovoltaic properties of solar cells based on multi-quantum well structures. A theoretical model including polarization effects is developed in order to evaluate the performance of the solar cells. The main contribution of this study consists in the improvement of the saturation current density due to polarization charges. In 
addition, the polarization effects on the solar cell photovoltaic characteristics are discussed and analyzed, in relation of the electron transport.

The paper is organized as follows. After a brief introduction, we present in Section 2 the current-voltage characteristics and photovoltaic parameters. Results are discussed in Section 3. Concluding remarks are in the last Section 3.

\section{Current-voltage characteristics and photovoltaic parameters}

\subsection{Theoretical framework}

The main assumptions used in our theoretical study are as follows:

i. Only radiative recombination is considered and the radiative coefficients are accounted for quantum wells and barrier epilayers.

ii. The semiconductors are non degenerate and so the MaxwellBoltzmann statics can be applied.

iii. Each quantum well as well as each barrier have respectively the same properties.

iv. The tkinesses of active layers are taken enough large, that makes tunneling effect negligible.

v. The spontaneous and piezoelectric polarizations are of intrinsic nature and no external strain is applied.

vi. The polarization charges are assumed to be uniformly distributed within an atomic layer.

The current-voltage relation of a MQW solar cell is expressed as $[7,19]$ :

$J(V)=J_{Q W 0}\left(1+\beta r_{R}\right)\left[\exp \left(\frac{q V}{n k T}\right)-1\right]-q \phi$

where $J_{Q W 0}$ is the saturation current density in the absence of polarization charges. $\beta$ is a parameter related to the intrinsic region, $r_{R}$ is the radiative enhancement. $q$ is the electron charge, $V$ is the voltage, $k T$ is the thermal energy and $\phi$ is the photo flux absorbed by the total quantum wells. The expression of $\beta$ and $r_{R}$ are given in [7].

\subsection{Saturation current}

The saturation current density without polarization charges is given by [19]:

$J_{Q W 0}=q n_{i B}^{2} \sqrt{\frac{4 \mu k T B_{B}}{q N}}$

where $N$ represents the doping density on the $\mathrm{n}$-and p-sides and is assumed to be $10^{18}\left(\mathrm{~cm}^{-3}\right), B_{B}$ is the recombination coefficient and $n_{i B}$ is the equilibrium intrinsic carrier concentration in the barrier epilayer. $\mu$ is the effective carrier mobility given by the electron mobility $\mu_{e}$ and the hole mobility $\mu_{h}$ according to [20]

$\mu=\frac{1}{4}\left(\sqrt{\mu_{e}}+\sqrt{\mu_{h}}\right)^{2}$

$\mu_{i}(N)=\mu_{\min , i}+\frac{\mu_{\max , i}-\mu_{\min , i}}{1+\left(\frac{N}{N_{g, i}}\right)^{\delta_{i}}}$

Recently, Zhang et al. [21,22] proposed a revised version of the saturation current as an attempt to explain the piezo-phototronic effects on the photovoltaic conversion in nano/microwire solar cells. In fact, it is the same result whether under the action of an applied external stress which is called the piezophototronic effect or internal stress provided by the lattice mismatch. This has allowed us to adopt the same approach as Zhang. In our study, the polarization $P(r)$ arises from two main sources: the spontaneous polarization due to the polar nature of the interface and the piezoelectric polarization due to the strain created by the lattice mismatch. Both of these lead to the appearance of a charge density at the interface. The discontinuity of $P$ between GaN and InGaN gives rise to polarization charge with an interfacial density $\sigma_{\mathrm{s}}$ such as $\sigma_{\mathrm{s}}=P$. Actually, the polarization charges are not distributed on a sheet but on a layer having a width $W_{p z}$. Indeed, the charges spread in the vicinity of the interface. Hereafter, we assume that the polarization charges are simulated by a bulk density

$\rho_{p}=\frac{P}{W_{p z}}$ for $0<z<W_{p z}$

Under these conditions, if we take into account the effects of polarization charges. Eq. (1) can be rewritten as:

$J(V)=J_{Q W}\left(1+\beta r_{R}\right)\left[\exp \left(\frac{q V}{n k T}\right)-1\right]-J_{p h}$

with:

$J_{Q W}=J_{Q W 0} \exp \left(\frac{q \rho_{p} W_{p z}^{2}}{2 \varepsilon V_{t h}}\right)$

For InGaN, the In-dependent spontaneous and piezoelectric polarizations are expressed according to [23]:

$P_{s p}(\operatorname{InGaN})=0.003 x+0.029$

$P_{p z}(\operatorname{InGaN})=-0.176 x$

The net polarization on the strained-InGaN/relaxed-GaN heterointerface, reads to:

$P=P_{s p}(\operatorname{InGaN})+P_{p z}(\operatorname{InGaN})-P_{s p}(G a N)$

\subsection{Photo current density}

The photocurrent density can be defined as [24]:

$J_{p h}=q N_{W} \sum_{n} N_{p h}\left(\lambda_{n}\right) \exp \left[-\alpha_{W}\left(\lambda_{n}\right) L_{W}\right] \Delta \lambda_{n}$

where $N_{W}$ is the number of quantum wells, $\Sigma_{n} N_{p h}\left(\lambda_{n}\right)$ is a parameter related to the solar spectrum, $n$ is the permitted transitions' wavelengh with line widths $\Delta \lambda_{n}, L_{W}$ is the width of the quantum well material and $\alpha(\lambda)$ is the absorption coefficient. The latter parameter depends not only on photon energy but on indium content. Several theoretical models have been proposed to describe the absorption coefficient $\alpha(\lambda)$ in the InGaN ternary. Brown et al. [12] propose the following relation to compute the absorption coefficient $\alpha(\lambda)$ :

$\alpha(\lambda)=\alpha_{0} \sqrt{a(x)\left(E-E_{g}\right)+b(x)\left(E-E_{g}\right)^{2}}$

with $E=\frac{h c}{\lambda}$ the photon energy.

The dimensionless fitting parameters $a(x)$ and $b(x)$ are given for small indium content [12]. For more details and to consider all the indium content compositions, we use further adjustment parameters $a_{f i t}$ and $b_{f i t}$. A linear interpolation is used to find the new fitting parameters over the entire composition range. In this case, the wavelength-dependent absorption coefficient has been determined for all alloy compositions. The parameters $a_{f i t}$ and $b_{f i t}$ are given using this linear interpolation: 


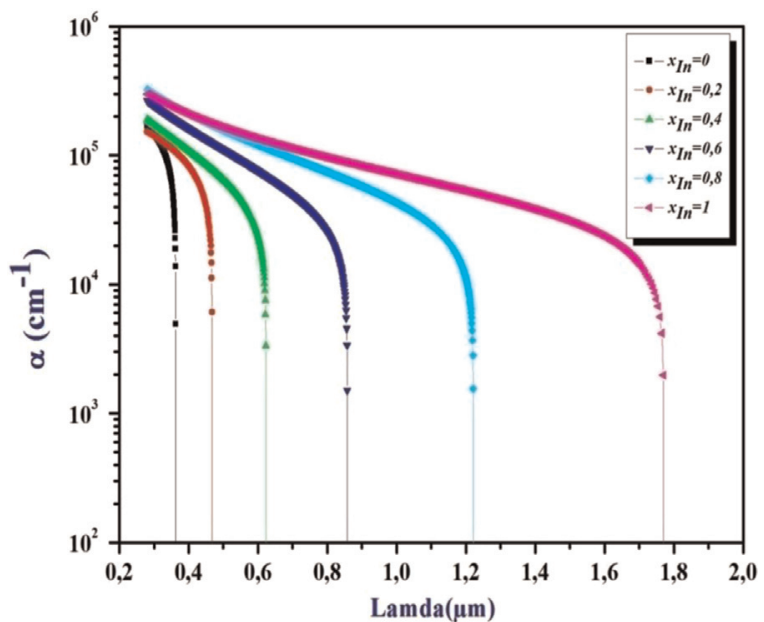

Fig. 1. Absorption coefficient of InGaN versus wavelength for different In compositions.

$a_{f i t}=12.87 x^{4}-37.79 x^{3}+40.43 x^{2}-18.35 x+3.52$

$b_{\text {fit }}=-2.92 x^{2}+4.05 x-0.66$

The collection efficiency $N(\lambda)$ is assumed to be constant.

Fig. 1 shows the absorption coefficient as calculated from the new fitting parameters for different indium contents in InGaN. The absorption coefficient $\alpha(\lambda)$ is of the order of $10^{5} \mathrm{~cm}^{-1}$ and drops rapidly below the band edge. It is revealed that the absorption spectrum can extend to longer wavelengths with the enhancement of indium composition in InGaN which is beneficial for the solar cells to absorb much enough of low energy photons.

\subsection{Photovoltaic parameters}

According to Eq. (1), by setting $J=0$, the open circuit voltage can be obtained as:

$V_{o c}=\frac{k T}{q} \ln \left(\frac{q \phi}{J_{Q W}}+1\right)$

For typical solar cells with $q \phi \gg J_{Q W}$, the open circuit voltage is approximately given by:

$V_{o c}=\frac{k T}{q}\left\{\ln \left(\frac{q \phi}{J_{Q W 0}}\right)-\frac{q \rho_{p} W_{p z}^{2}}{2 \varepsilon V_{t h}}\right\}$

Let $P_{m}=V_{m} I_{m}$ and $P_{m}$ be respectively the maximum converted power and the total incident solar power. Thus, the fill factor and the external quantum efficiency of the photovoltaic cell are defined as:

$\eta=\frac{P_{\max }}{P_{\text {inc }}}=\frac{V_{o c} F F J_{p h}}{P_{\text {inc }}}$

where $P_{\text {inc }}$ is the incident irradiance per unit area in $\mathrm{mW} / \mathrm{cm}^{2}$.

While the fill factor is taken under the form:

$F F=\frac{I_{m} V_{m}}{P_{\text {inc }}}$

\section{Results and discussion}

The photovoltaic solar cell to model consists in InGaN/GaN
Solar radiations

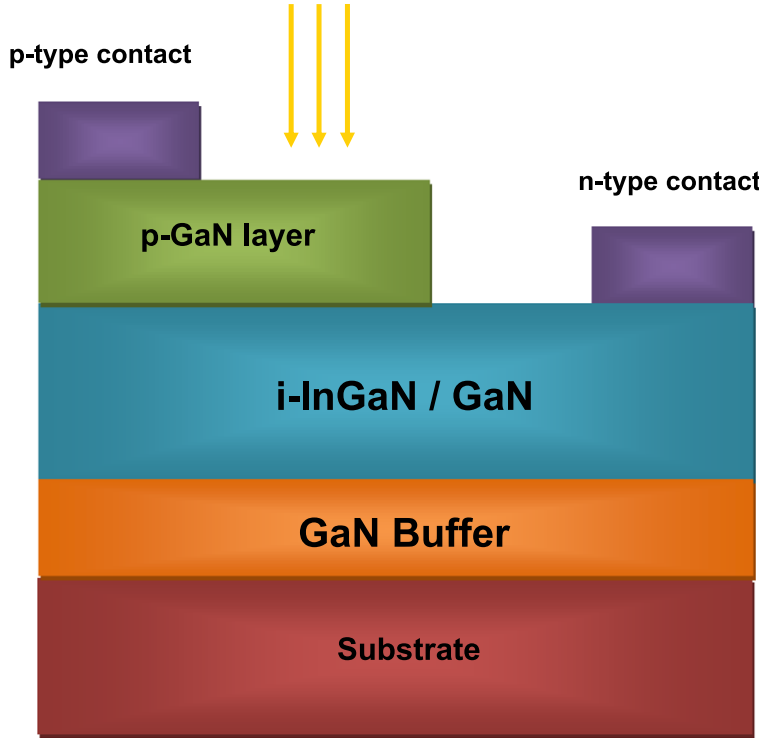

Fig. 2. Schematic cross section of the InGaN/GaN MQW solar cell to model.

MQWs sandwiched between p-type and n-type GaN contacts (see Fig. 2). The thickness of the barriers is fixed at $10 \mathrm{~nm}$ whereas the width of the QWs is $5 \mathrm{~nm}$. The polarization charges are assumed to be spreaded within $W_{p z}$. The solar cell is illuminated under $\mathrm{AM}_{1.5}$. The In composition is treated as a parameter.

To investigate the polarization effects of electrical properties of InGaN/GaN, the power density voltage curves are first simulated with and without polarization as shown in Fig. 3. The solar cell contains only a single $\mathrm{QW}$ for indium concentration of 0.6 . The same curves are plotted for several values of indium content $x$. Then, we determine the maximal value of the power $P_{\max }$ for a given value of $x$. We observe that the greatest value of $P_{\max }$ is obtained for $x=0.6$. For this reason, we chose this particular value to plot Fig. 3. It clearly appears from the relevant results that the presence of polarization charges increases significantly the power density. Due to lack of experimental data for simulated structures, we intend to compare our results to that of $\mathrm{p}-\mathrm{GaN} / \mathrm{n}-\mathrm{In}_{0.2} \mathrm{Ga}_{0.8} \mathrm{~N}$ heterojunction solar cells studied by Mehta et al. [26]. They have incorporated the polarization into a source code of PC1D. They observed an increase in power achieving 38 percent. The beneficial

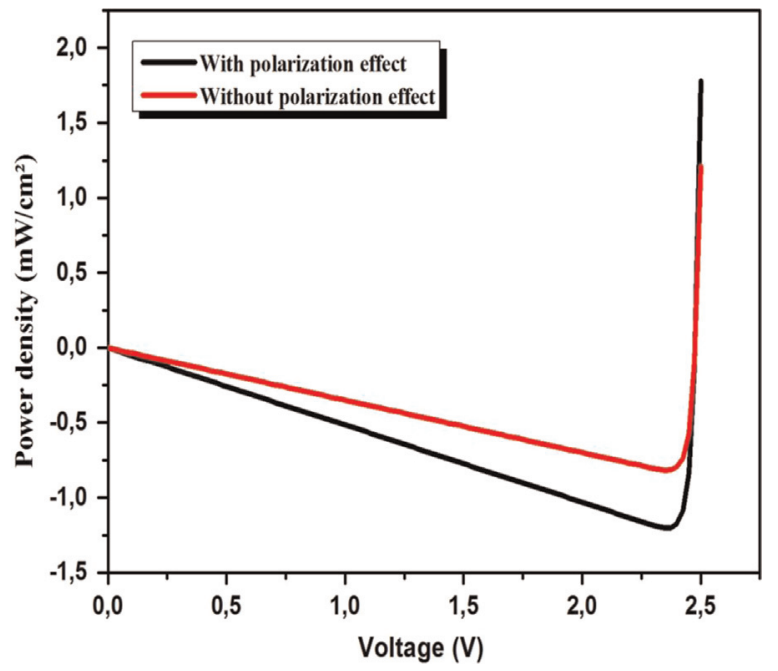

Fig. 3. Power density as calculated versus voltage without and with polarization effects. 


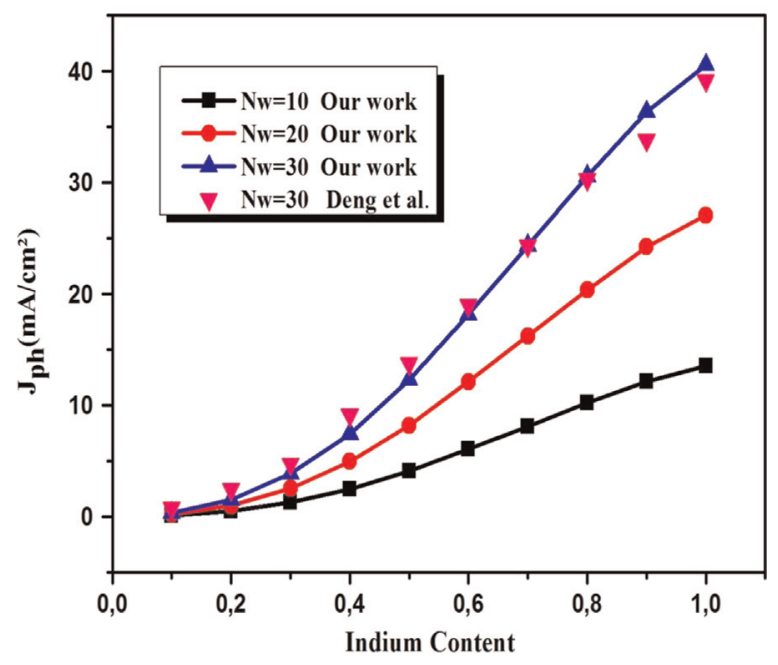

Fig. 4. Dependence of $J_{p h}$ on indium composition and the number of quantum wells.

aspect of the polarization charges on the power density has also been observed by Jih et al. [25] who simulated the GaN/InGaN double heterostructures in the $\mathrm{N}$-face by a comparison $\mathrm{J}-\mathrm{V}-\mathrm{P}$ characteristics in the situations of no polarization and reversed polarization.

To explain the effect of polarization charge on the output power, we have calculated the saturation current; with and without polarization charges. Eq. (4) presents that the saturation current $J_{Q W}$ is an exponential function of local polarization charges. It is clear that the current is directly related to the polarization charges.

Jih et al. [25] demonstrate numerically, using the Abbreviation of Advanced Physical Models of Semiconductor Devices (APSYS), that in the case of InGaN p-i-n solar cell with $\mathrm{N}$ face configuration, the presence of polarization charges increases significantly the power density but they also found that the photocurrent density is not affected by this charges, so we can say that the increase of the power density is mainly due to the increase of saturation current.

Fig. 4 exhibits the dependence of Jph on In composition as well as the number $N_{w}$ of QWs. It is revealed that the photocurrent density strongly depends on indium content. In fact, the current density depends mainly on the number of electron-hole pairs created by absorbed photons. Indeed, photons with energies higher than the p-GaN band gap are absorbed by the top p-GaN whereas photons with energy higher than InGaN band gap are absorbed in the intrinsic region, which explains the dependence of $J_{p h}$ on the indium content. On the other hand, a linear increase of $J_{p h}$ versus $N_{w}$ is observed. An increase in the number of quantum wells indeed provides more absorption photons. This can explain that the increment of $N_{W}$ linearly enhances $J_{p h}$. We thus conclude that the $J_{p h}$ may be controlled by two main factors: the indium content and the number of QWs. Fig. 4 also shows that our results agree with that found by Deng et al. [7].

Fig. 5 presents the dependence of the open circuit voltage $V_{o c}$ on In composition for a MQW. Contrary to $J_{p h}, V_{o c}$ drops with the increasing of indium content. In order to analyze the piezoelectric effect on the $V_{o c}$, we have compared the $V_{o c}$ with and without polarization effect. The figure reveals that the piezoelectric charges do not have a significant influence on the $V_{o c}$. Mehta et al. [26] have studied the effect of polarization charges on the open circuit voltages in $\mathrm{p}-\mathrm{GaN} / \mathrm{n}-\mathrm{In}_{0.2} \mathrm{Ga}_{0.8} \mathrm{~N}$ heterostructure. They found that the $V_{o c}$ for both cases with and without polarization is compared at around $2.2 \mathrm{~V}$.

Finally, the In composition-dependent conversion efficiency for

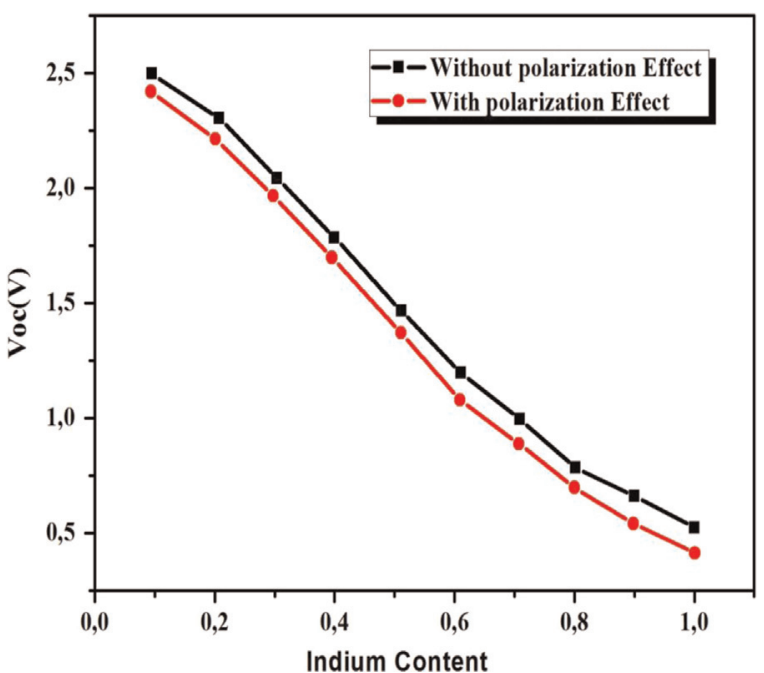

Fig. 5. Dependence of $V_{o c}$ as a function of In composition for 30 periods of quantum wells.

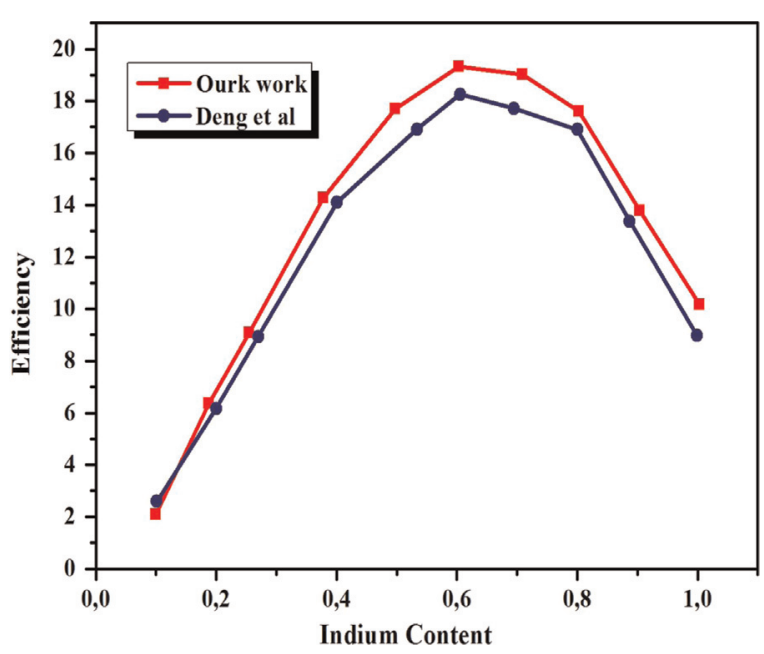

Fig. 6. Calculation of conversion efficiency versus In composition for 30 periods of quantum wells.

a 30 MQWs is depicted in Fig. 6. As can be seen, the optimal conversion efficiency is achieved for an In composition $x=0.6$. It should be noted that when the number of QWs is changed, the behavior of the efficiency versus $x$ remains the same and exhibits a maximum for $x=0.6$. However, the optimal value of the efficiency depends linearly on $N_{W}$.

The presence of an optimal composition is expected since the indium content $x$ plays antagonistic effects on $J_{p h}$ and $V_{o c}$. Our results agree well with that obtained by Deng et al. [7] who have neglected the polarization effects. The effect of polarization charges on the efficiency of a solar cell is noticeable. At this stage, we can say that the performance of the solar cells cannot be properly assessed by neglecting the polarization charges.

\section{Conclusion}

A new model to simulate InGaN/GaN multiple quantum well solar cells has been elaborated. This model takes into account the piezoelectric polarization. The revision has been made both on saturation current and open circuit voltage $V_{o c}$. It is found that the spontaneous and piezoelectric polarizations strongly affect the saturation current as well as the output power. They, however, do 
not have significant influence on the open circuit voltage $V_{o c}$. This model has been applied successfully to investigate the photovoltaic characteristics of the solar cells taking into account the polarization effects. Obtained results would be helpful for designing high performance photovoltaic solar cells based on the InGaN/GaN system.

\section{References}

[1] Y. Jing, Z.D. Gang, J.D. Sheng, L.Z. Shun, C. Ping, L. Liang, W.L. Liang, L.L. Jing, H. X. Jing, H.X. Guang, W. Hui, Z.J. Jun, Z.S. Ming, Z.B. Shun, Y. Hui, Chin. Phys. B 23 (2014).

[2] L. Wang, W. Hu, X. Chen, W. Lu, J. Electron. Mater. 41 (2012) 2130-2138.

[3] C.J. Neufeld, N.G. Toledo, S.C. Cruz, M. Iza, S.P. DenBaars, U.K. Mishra, Appl. Phys. Lett. 93 (2008).

[4] C.Y. Liu, C.C. Lai, J.H. Liao, L.C. Cheng, H.H. Liu, C.C. Chang, G.Y. Lee, J.I. Chyi, L. K. Yeh, J.H. He, T.Y. Chung, L.C. Huang, K.Y. Lai, Thin Solid Films 529 (2013) $278-281$.

[5] J. Wu, J. Appl. Phys. 106 (2009) 01110101-1.

[6] M.E. Levinshtein, S.L. Rumyantsev, M.S. Shur, Properties of Advanced Semiconductor Materials, John Wiley and Sons, New York, 2001.

[7] O. Deng, X. Wang, H. Xiao, C. Wang, H. Yin, H. Chen, Q. Hou, D. Lin, J. Li, Z. Wang, X. Hou, J. Phys. D: Appl. Phys. 44 (2011) 265103.

[8] A.T.M. Golam Sarwar, R.C. Myers, Appl. Phys. Lett. 101 (2012) 143905.
[9] J.Y. Chang, S.H. Yen, Y.A. Chang, Y.K. Kuo, IEEE J. Quantum Electron. 49 (2013).

[10] V. Fiorentini, F. Bernardini, O. Ambacher, Appl. Phys. Lett. 80 (2002) 1204-1206.

[11] O. Ambacher, J. Majewski, C. Miskys, A. Link, M. Hermann, M. Eickhoff, M. Stutzmann, F. Bernardini, V. Fiorentini, V. Tilak, B. Schaff, L.F. Eastman, J. Phys. Condens. Matter, 14, (2002) 3399-3434.

[12] G.F. Brown, J.W. Ager III, W. Walukiewicz, J. Wu, Sol. Energy Mater. Sol. Cells 94 (2010) 478-483.

[13] J.Y. Chang, Y.K. Kuo, J. Appl. Phys. 112 (2012).

[14] V. Gorge, A.M. Dubois, Z. Djebbour, K. Pantzas, S. Gautier, T. Moudakir S. Suresh, A. Ougazzaden, Mater. Sci. Eng. B 178 (2013) 142-148.

[15] X.M. Cai, S.W. Zeng, B.P. Zhang, Appl. Phys. Lett. 95 (2009) 173504

[16] X.M. Cai, S.W. Zeng, B.P. Zhang, Electron. Lett. 24 (2009) 1266.

[17] A.T.M. Golam Sarwar, R.C. Myers, Appl. Phys. Lett. 101 (2012) 143905.

[18] Z.Q. Li, M. Lestradet, Y.G. Xiao, S. Li, Phys. Status Solidi A 208 (4) (2011) 928-931.

[19] N.G. Anderson, J. Appl. Phys. 78 (3) (1995).

[20] T.T. Mnatsakanov, M.E. Levinshtein, L.I. Pomortseva, S.N. Yurkov, G.S. Simin, M. A. Khan, Solid-state Electron. 47 (2003) 111-115.

[21] Y. Zhang, Y. Liu, Z.L. Wang, Adv. Mater. (2011) 1-10.

[22] Y. Zhang, Y. Yang, Z.L. Wang, Dyn. Artic. 5 (2012) 6850-6856.

[23] H.W. Wang, P. Yu, IEEE J. Photovolt. 3 (2013) 2156-3381.

[24] S.J. Lade, A. Zahedi, Microelectron. J. 35 (2004) 401.

[25] J.Y. Chang, Y.K. Kuo, J. Appl. Phys. 112 (2012).

[26] M. Mehta, O. Jani, C. Honsberg, B. Jampana, I. Ferguson, A. Doolittle, 22nd European Photovoltaic Solar Energy Conference, vol. 3-7, 2007. 